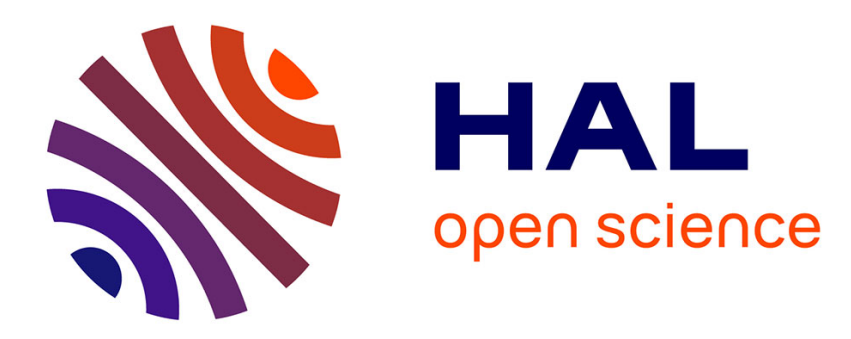

\title{
Chambre à étincelles sonique pour électrons de basse énergie
}

G. Metzger, M. Riedinger, F. Schmitt, J. Gresser, G. Sutter

\section{To cite this version:}

G. Metzger, M. Riedinger, F. Schmitt, J. Gresser, G. Sutter. Chambre à étincelles sonique pour électrons de basse énergie. Revue de Physique Appliquée, 1969, 4 (2), pp.307-309. 10.1051/rphysap:0196900402030700 . jpa-00243275

\section{HAL Id: jpa-00243275 https://hal.science/jpa-00243275}

Submitted on 1 Jan 1969

HAL is a multi-disciplinary open access archive for the deposit and dissemination of scientific research documents, whether they are published or not. The documents may come from teaching and research institutions in France or abroad, or from public or private research centers.
L'archive ouverte pluridisciplinaire HAL, est destinée au dépôt et à la diffusion de documents scientifiques de niveau recherche, publiés ou non, émanant des établissements d'enseignement et de recherche français ou étrangers, des laboratoires publics ou privés. 


\title{
GHAMBRE A ÉTINGELles SONIQUE POUR ÉLEGTRONS DE BASSE ÉNERGIE
}

\author{
G. METZGER, M. RIEDINGER, F. SGHMITT, J. GRESSER et G. SUTTER, \\ Institut de Recherches Nucléaires, Strasbourg.
}

Résumé. - Il a été réalisé un spectromètre magnétique à chambres à étincelles soniques pour paires d'électrons de conversion. On décrit l'appareillage et on donne les résultats de l'étude de l'ensemble sonique (localisation à $0,06 \mathrm{~mm}$ près dans une surface utile de $75 \mathrm{~cm}^{2}$ ).

Abstract. - In order to measure conversion electron pairs a magnetic spectrometer has been constructed, using sonic spark chambers. This paper describes the sonic circuit and its performance (spark located at $\pm 0.06 \mathrm{~mm}$ in a $75 \mathrm{~cm}^{2}$ area).

I. Introduction. - En vue d'étudier le spectre et la corrélation de paires d'électrons de conversion (basses énergies), nous avons réalisé un appareil semiautomatique comprenant un aimant et des chambres à étincelles à localisation sonique. Le rôle de l'aimant est de séparer les électrons des positrons et de définir en fonction de l'énergie une trajectoire. Les chambres à étincelles permettent de reconstituer cette trajectoire. Actuellement, les diverses parties sont étudiées et l'ensemble de l'appareillage est en cours de mise au point. Nous décrivons ici plus particulièrement l'ensemble sonique.

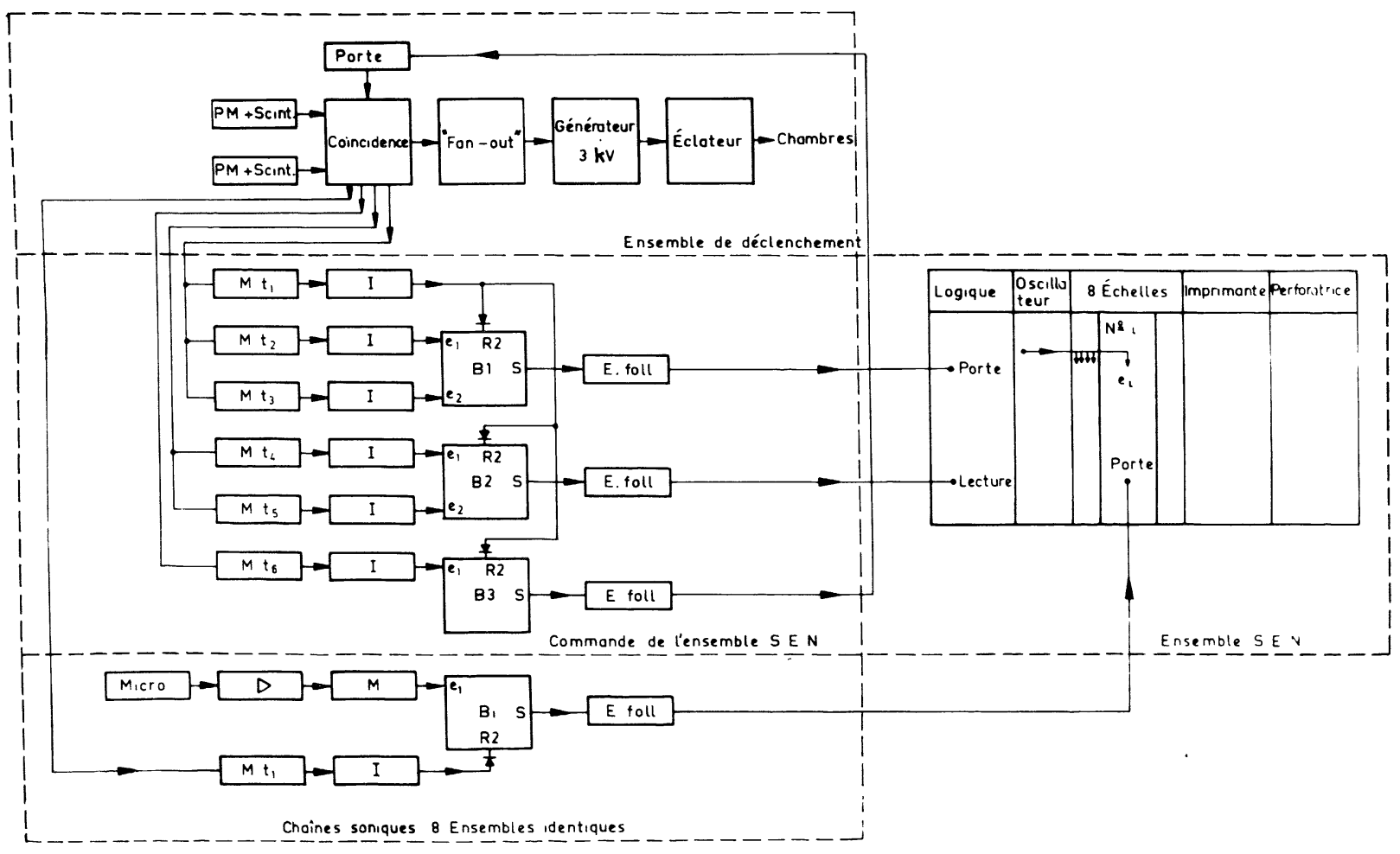

FIG. 1. 
II. Dispositif expérimental. - II.1. Les Chambres A ÉTincelles. - Les impératifs qui ont présidé à la conception de ces chambres sont une absorption minimale et une localisation permettant une sortie rapide des informations sous une forme directement utilisable par une calculatrice. La première de ces raisons a fait choisir des chambres à faible interplaque $(5 \mathrm{~mm})$ dont les électrodes sont des feuilles minces d'acier inoxydable $(12,5 \mu \mathrm{m})$. Elles sont étanches pour avoir une absorption minimale des particules, tout en permettant le choix d'un gaz de remplissage relativement dense (néon) à la pression atmosphérique. Elles sont circulaires (diamètre utile $10 \mathrm{~cm}$ ) pour obtenir par sertissage une tension uniforme des feuilles d'acier inoxydable. Une pièce en plexiglas maintient l'écartement des plaques, l'étanchéité étant assurée par des fenêtres en mylar $(6 \mu \mathrm{m})$. Cette même pièce supporte les microphones de mesure. Quatre microphones tubulaires en céramique piézoélectrique captent le son produit par l'étincelle; le temps-de-vol étant directement lié à la distance, nous obtenons la position de l'étincelle [2]. Le délai apporté par les circuits de déclenchement est de l'ordre de 60 ns (P.M. inclus).

II .2. Ensemble sonique ( fig. 1) [3]. — L'ensemble de localisation de l'étincelle comprend essentiellement trois parties : i) l'ensemble SEN d'enregistrement (SEN 300 complet); ii) la chaîne sonique; iii) la commande de l'ensemble SEN. Les oscillogrammes corrélatifs de la figure 2 montrent le fonctionnement. Au temps $t_{0}$, les échelles sont à zéro, toutes les portes de comptage fermées. Une étincelle se produit. Pendant le temps $t_{1}(47 \mu \mathrm{s})$, tous les bistables sont maintenus à zéro de façon à éviter la perturbation due au rayonnement de l'étincelle. Puis le bistable $B_{1}$ de commande de porte générale des échelles est ouvert au bout du temps $t_{2}(62,25 \mu \mathrm{s})$. Les échelles comptent les impulsions de l'oscillateur. Le temps $t_{2}$ constitue donc un temps mort à rajouter au contenu des échelles. Au bout de $t_{\mathrm{i}}$, le ième microphone reçoit le son et le bistable $B_{i}$ ferme la porte de comptage de l'échelle $\mathrm{n}^{0}$ i. Celle-ci contient donc un chiffre proportionnel au temps-de-vol du son pour le microphone $n^{\circ}$ i. Enfin, au bout de $t_{3}(512 \mu \mathrm{s})$ correspondant à la distance maximale pouvant être parcourue par le son, $B_{1}$ ferme la porte générale des échelles. Ainsi, même si l'un des microphones n'a pas répondu, on peut passer à l'enregistrement. Cet ordre est donné par $\mathrm{B}_{2}$ au bout du temps $t_{4}(513 \mu \mathrm{s})$ jusqu'à $t_{5}(530 \mu \mathrm{s})$. La logique SEN lit alors le contenu des échelles et l'imprime en clair par l'intermédiaire d'une imprimante Hewlet-Packard et, en même temps, perfore une bande selon le code BCD sur une perforatrice Tally. Durant tout ce temps $\left(T_{6}=5 \mathrm{~s}\right)$, une porte sur l'alimentation du circuit de coïncidences est fermée par le bistable $B_{3}$, empêchant la venue d'une nouvelle information pendant le traitement de la première. L'ensemble de toutes ces opérations se fait au plus en cinq secondes, cette durée étant donnée essen- tiellement par la cadence d'impression (en parallèle) ou de perforation (en série). Des adjonctions commercialisées sur l'ensemble SEN permettraient une cadence

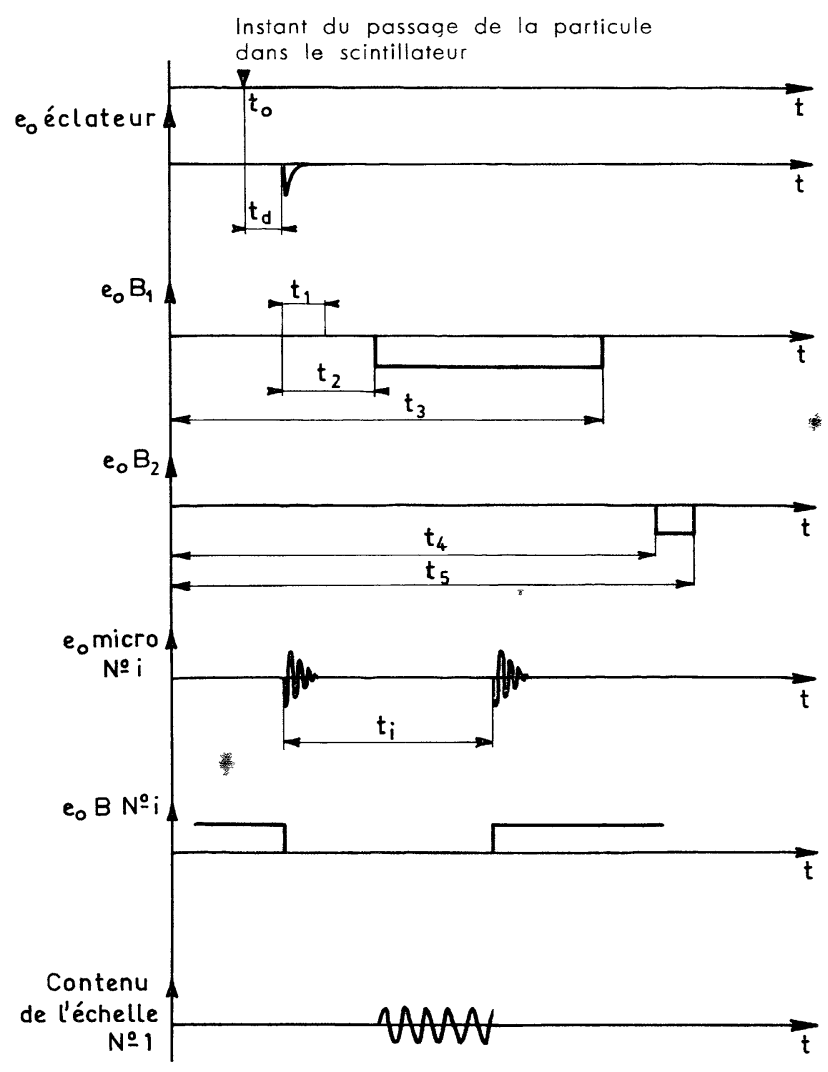

FIG. 2.

beaucoup plus rapide, et même le branchement « on line » de l'appareillage.

Pour la mesure de la précision de localisation, une électrode est percée d'une série de trous $(\varnothing<0,1 \mathrm{~mm})$ régulièrement espacés. Un seul de ces trous reçoit le rayonnement U.V. d'un éclateur déclenché environ 100 ns avant la chambre. Les photoélectrons émis par les bords du trou sont alors à l'origine de l'étincelle dans la chambre. Cette étincelle se produit donc à un endroit précis prédéterminé.

La précision de localisation sonique ainsi relevée $( \pm 0,06 \mathrm{~mm})$ est limitée par le temps de montée du signal observé au microphone $(\simeq 0,8 \mu$ s) combiné après amplification au seuil de déclenchement du monostable $\mathrm{M}$ de la chaîne sonique $(0,5 \mathrm{~V})$. La linéarité de la détection est meilleure que $1 \%$ (précision de mesure) sur toute la superficie utile de la chambre. Ces caractéristiques sont dues, d'une part, à la faible superficie de la chambre qui implique la détection $\mathrm{du}$ front de choc du signal avant sa détérioration $(c=466 \mathrm{~m} / \mathrm{s}$ au lieu de $\simeq 300 \mathrm{~m} / \mathrm{s})$, et, d'autre part, à la grande qualité de l'amplificateur à circuit intégré. 
Les principales difficultés rencontrées se situent dans l'élimination des déclenchements parasites sur le rayonnement de l'étincelle, et dans la mise au point de l'amplificateur de la chaîne sonique : gain 8000 , temps de montée pour un signal de $1 \mathrm{mV}$ saturant l'amplificateur, inférieur à la microseconde (bande passante équivalente à $1 \mathrm{MHz}$ ); les fréquences basses (enveloppe du signal) doivent être transmises.

\section{BIBLIOGRAPHIE}

[1] Metzger (G.) et al., Nucl. Instr. Methods, 1967, 47, 100, 52, 331; 1968, 48, 315.

[2] MAGLiG (B. C.) et KIRSTEN (F. A.), Nucl. Instr. Methods, 1962, 17, 49.

[3] BiZARD (G.) et al., Nucl. Instr. Methods, 1966, 45, 119. 